\title{
Efficacy of MRP8/14 as a Marker of Disease Activity in Rheumatoid Arthritis
}

\author{
Tetsuro Yamasaki', Ryo Oda1, Kan Imai'1, Daigo Taniguchi', Shogo Toyama1, \\ Takahiro Seno'2, Yuji Arai1, Kazuya Ikoma1, Hiroyoshi Fujiwara1, Daisaku Tokunaga1, \\ Yutaka Kawahito², Toshikazu Kubo ${ }^{1}$ \\ ${ }^{1}$ Department of Orthopaedics, Graduate School of Medical Science, Kyoto Prefectural University of Medicine, \\ Kyoto, Japan \\ ${ }^{2}$ Department of Inflammation and Immunology, Graduate School of Medical Science, Kyoto Prefectural \\ University of Medicine, Kyoto, Japan \\ Email: vama-fe@koto.kpu-m.ac.jp
}

Received 11 April 2016; accepted 24 May 2016; published 27 May 2016

Copyright () 2016 by authors and Scientific Research Publishing Inc.

This work is licensed under the Creative Commons Attribution International License (CC BY).

http://creativecommons.org/licenses/by/4.0/

(c) (i) Open Access

\section{Abstract}

Objective: Early and accurate evaluation of the presence and activity of synovitis is extremely important in the diagnosis and treatment of rheumatoid arthritis. Myeloid related protein 8/14 (MRP8/14), also known as calprotectin or $\$ 100 A 8 / A 9$ is considered as a sensitive marker for local inflammatory activity in rheumatoid arthritis. The aim of this study is to demonstrate the efficacy of MRP8/14 as a marker of disease activity in RA. Methods: Thirty-one patients with diagnosis of RA who received treatment without biological drugs at our institution were included in this study. Serum MRP8/14, CRP and MMP-3 were tested in all patients. Disease activity was evaluated using DAS28-CRP and SDAI. Ultrasonography was performed on the wrists and MCP joints of both hands using semi-quantitative scale of power Doppler signal. The sum of scales in joints was calculated as the PD score. The correlation of MRP8/14 with serum biomarkers, disease activity and ultrasonography examination was investigated. Result: Serum MRP8/14 was strongly correlated with CRP $(r=0.63)$ and MMP-3 $(r=0.69)$. A correlation was observed between serum MRP8/14 and DAS28-CRP ( $r=0.53$ ) and SDAI $(r=0.66)$. No significant correlation was found between PD scores and MRP8/14. Conclusion: This study demonstrated that MRP8/14 is correlated with evaluated disease activity and markers of serum inflammatory response in patients not using biological drugs. MRP8/14 is considered an effective new method for objective evaluation of synovitis in RA.

\section{Keywords}

Rheumatoid Arthritis, Myeloid Related Protein 8/14 (MRP8/14), Calprotectin, Disease Activity 


\section{Introduction}

Rheumatoid arthritis (RA) is an autoimmune inflammatory disease which mainly causes inflammation of the joint synovium. Prolonged synovitis leads to progression of joint destruction and joint degeneration. Pain due to synovitis and limitations in the range of joint motion due to joint degeneration greatly limit patients' activities of daily living (ADL). In the early phase of RA, a period during which significant therapeutic efficacy can be expected by therapeutic intervention (the window of opportunity) exists. Once joint destruction has occurred, operative therapy is often needed for functional recovery and pain relief. Therefore, early intervention and adequate synovitis control are necessary to prevent joint destruction. Thus, early and accurate evaluation of the presence and activity of synovitis is extremely important in the diagnosis and treatment of rheumatoid arthritis.

Disease Activity Score (DAS) 28 and Simplified Disease Activity Index (SDAI) are currently used for evaluation of disease activity in RA. Each of these is a composite disease activity score that is a quantitative evaluation consisting of evaluation by an examiner, the patient's subjective evaluation and serum inflammatory response as an objective measure. Use of the DAS28 and SDAI improves control of RA [1]-[3], and is effective in determining whether treatment with a biological drug is necessary [4]. However, certain challenges remain, as for example, joint destruction may progress even in cases classified as remission by DAS28. In addition, although the use of joint ultrasonography and contrast-enhanced MRI for the evaluation of localized synovitis has been growing, the validity of joint ultrasonography evaluation may depend on the examiner's experience, and contrast-enhanced MRI has a high cost.

Myeloid related protein (MRP) 8/14 has attracted attention in recent years as an index of synovitis activity in rheumatoid arthritis patients. MRP8/14 is a calcium-binding protein belonging to the S100 protein family which is also known as calprotectin [5] [6] or S100A8/A9 [7]. MRP8 and MRP14 have an important role in the acute inflammatory reaction [5] [8] [9], as they function to mobilize inflammatory cells to the inflamed region [10]. MRP8/14 is released from activated granulocytes or mononuclear cells and macrophages which are present in the synovial membrane and joint fluid [11] [12]. C-reactive protein (CRP) is mainly produced in hepatocytes through the action of interleukins (tumor necrosis factor [TNF], interleukin [IL]-1 and IL-6). It has been reported that this process is affected by genetic factors [13]. On the other hand, MRP8/14 directly reflects the leukocyte counts within inflamed joints. Thus, MRP8/14 is considered a sensitive marker for localized inflammatory activity in RA [11] [12] [14].

We hypothesized that if the relationship between MRP8/14 as an index of synovitis and existing RA disease activity evaluation methods was adequately clarified, easy and objective evaluation of the RA condition using MRP8/14 would be feasible.

The purpose of this study is to demonstrate the efficacy of MRP8/14 as a marker of disease activity in RA.

\section{Patients and Methods}

\subsection{Patient Characteristics}

This study was conducted with the approval of the Ethical Review Board at our institution (RBMR-C-976-1). Out of the 106 patients with a diagnosis of rheumatoid arthritis based on the 1987 American College of Rheumatology (ACR) criteria [15] who received treatment without biological agents at our institution, and who were not using biological drugs, 31 patients ( 3 male and 28 female) from whom written consent was obtained were included in this study. The patient characteristics were shown in Table 1. Mean age was $62.2 \pm 1.6$ years, and mean disease duration was $141.1 \pm 26.3$ months. Twenty-eight patients used methotrexate with mean dosage of $7.10 \pm 0.67 \mathrm{mg}$. One patient used prednisolone at $4 \mathrm{mg} / \mathrm{day}$. Four patients used tacrolimus at 0.5 to $2 \mathrm{mg} / \mathrm{day}$ (mean $1.1 \mathrm{mg} /$ day).

\subsection{Methods}

CRP was measured as existing biomarkers of synovitis. In addition, matrix metalloproteinase-3 (MMP-3) was measured as a biomarker of cartilage destruction. Latex coagulating nephelometry was used to measure CRP and MMP-3. MRP8/14 concentration was assessed using a commercial sandwich enzyme-linked immunosorbent assay system (ELISA) development kit (BMA Biomedicals, Rheinstrasse, Switzerland). The specific monoclonal antibody for the MRP8/14 heterodimer (mAb 27E10) was used as primary antibody and a polyclonal antibody coupled with horseradish peroxidase was used as secondary antibody. The correlation of serum 
Table 1. Characteristics of patients at enrollment in this study.

$\begin{array}{cc}\text { Number of patients } & 31 \\ \text { Age (year) } & 62 \\ \text { Gender (\% female) } & 90.3 \\ \text { Disease duration (months) } & 141 \\ \text { Using methotrexate (\%/mg) } & 90 / 7.1 \\ \text { CRP (mg/ml) } & 0.63 \\ \text { MMP-3 (mg/dl) } & 97.0 \\ \text { MRP8/14 (ng/dl) } & 6630.3 \\ \text { DAS28-CRP (remission/low/moderate/severe) (\%) } & 46.4 / 25.8 / 22.6 / 3.2 \\ \text { SDAI (remission/mild/moderate/severe) (\%) } & 32.2 / 35.5 / 29.0 / 3.2 \\ \text { Number of swollen joints } & 1.6 \\ \text { Number of tender joints } & 1.5 \\ \text { Visual analog scale for patient pain (mm) } & 21.4 \\ \text { Power Doppler score } & 5.6\end{array}$

MRP8/14 with CRP, and MMP-3 was evaluated.

Rheumatology specialist evaluated swelling and tenderness in 28 joints, and the physician's global assessment score. In addition, pain was evaluated using the Visual analogue scale (VAS) on a 100-mm VAS. Disease activity was evaluated using the DAS28-CRP [16] and the SDAI [17]. In DAS28-CRP, a score of less than 2.3 was defined as remission, a score of 2.3 to 3.2 was defined as low disease activity, a score of 3.3 to 5.1 was defined as moderate disease activity, and a score greater than 5.1 was defined as high disease activity. In SDAI, a score of less than 3.3 was defined as remission, a score of 3.3 to 11 was defined as mild disease activity, a score of 11 to 26 was defined as moderate disease activity, and a score greater than 26 was defined as severe disease activity. The relationship between DAS28-CRP and SDAI, and serum MRP8/14 was statistically evaluated.

Ultrasonography was performed on the wrists (radiocarpal joint, intercarpal joint, ulnocarpal joint) and metacarpophalangeal (MCP) joints of both hands. The intra-articular power Doppler (PD) signal was subjectively graded on a semi-quantitative scale ranging from 0 to 3 [18]. The sum of grades in 16 joints was calculated as the PD score. Therefore, the PD score ranged from 0 to 48. All ultrasonography examinations were performed using HI VISION Avius, (Hitachi Aloka Medical, Tokyo, Japan) with a 3 - $15 \mathrm{MHz}$ convex probe (Hitachi Aloka). Correlations between PD scores and serum MRP8/14 values were investigated.

SPSS Statistics Version 21 (Chicago, IL, USA) was used for the statistical analysis. Quantitative values were shown as mean \pm standard error. The correlation between MRP8/14 and clinical evaluation and hematological tests data was investigated using Pearson's correlation analysis. P $<0.05$ was defined as statistically significant. The relationship between PD scores from hand joint ultrasonography examination and MRP8/14, DAS28-CRP, and SDAI was investigated using Spearman signed rank test.

\section{Results}

A strong correlation was observed between MRP8/14 and CRP, the serum markers of inflammatory response and MMP-3, a marker of cartilage destruction (Table 2).

According to the evaluation using DAS28-CRP, remission was observed in 15 cases (48.4\%), low disease activity in 8 cases (25.8\%), moderate disease activity in 7 cases (22.6\%), and severe disease activity in 1 case (3.2\%). According to the evaluation using SDAI, remission was observed in 10 cases (32.3\%), mild disease activity in 11 cases (35.5\%), moderate disease activity in 9 cases (29.0\%), and severe disease activity in 1 case (3.2\%).

A correlation was observed between serum MRP8/14 and DAS28-CRP and SDAI. In addition, MRP8/14 was correlated with each of the clinical evaluation components including swollen joint count (SJC) and VAS for patient pain (Table 3).

The mean PD score from joint ultrasonography was $4.4 \pm 1.3$. No significant correlation was found between 
Table 2. Correlations between MRP8/14 and laboratory tests.

\begin{tabular}{ccc}
\hline & CRP & MMP-3 \\
\hline Correlation coefficients & 0.63 & 0.69 \\
P values & $\mathrm{P}<0.001$ & $\mathrm{P}<0.001$
\end{tabular}

Serum MRP8/14 strongly correlated with CRP and MMP-3.

Table 3. Correlations between MRP8/14 and clinical assessments.

\begin{tabular}{cccccc}
\hline & SDAI & DAS28-CRP & SJC & TJC & Patient pain VAS \\
\hline Correlation coefficients & 0.66 & 0.53 & 0.40 & 0.25 & 0.59 \\
P values & $\mathrm{P}<0.001$ & $\mathrm{P}<0.01$ & $\mathrm{P}<0.05$ & N.S. & $\mathrm{P}<0.001$ \\
\hline
\end{tabular}

There was correlation between MRP8/14 and disease activity assessed with SDAI and DAS28-CRP.

PD scores and MRP8/14. Moreover, no significant correlation was found between PD scores and either DAS28CRP or SDAI.

\section{Discussions}

Evaluation of synovitis activity in RA treatment is extremely important for determining the initiation of RA treatment and selecting or changing medication. Adequate control of synovitis activity connects to the prevention of joint destruction, and therefore to the prevention of a decrease in ADL and QOL.

Sunahori et al. compared MRP8/14 levels in joint fluid between osteoarthritis patients and RA patients, and demonstrated that MRP8/14 levels are significantly higher in RA patients [19]. Kane et al. demonstrated that MRP8/14 expression is increased in macrophages in the inflamed synovial membrane, and MRP8/14 in joint fluid of RA patients correlates with serum MRP8/14. These findings suggest that MRP8/14 is suitable for the evaluation of synovitis due to RA.

In previous studies, a relationship has been reported between MRP8/14 and disease activity [20] [21], and the evaluation of localized synovitis [20] [21], or more specifically a relationship with joint destruction observed with plain radiography [21] [22] in rheumatoid arthritis patients. Moreover, a relationship has been suggested between MRP8/14 and serum inflammatory response biomarkers including CRP, and between MRP8/14 and various autoantibodies, such as anti-CCP antibody [20] [23].

In this study, MRP8/14 was correlated with CRP, MMP-3, and disease activity assessed with DAS28-CRP and SDAI. In addition, MRP8/14 was also correlated with the swollen joint count (SJC) and VAS for patient pain, which are elements of these composite disease activity scores. DAS28 and SDAI make easy evaluation possible in the clinical setting. As the development of these evaluation methods has improved control of rheumatoid arthritis, they are currently widely used in evaluating RA disease activity. However, as evaluation of SJC and TJC is relatively strongly subjective, it is considered that there are issues with reproducibility between examiners [24]. Therefore, inter-examiner error is likely to be generated in DAS28 and SDAI including those evaluations. Since MRP8/14 reflects both objective and subjective evaluation endpoints, the possibility of evaluating RA disease activity more objectively and quantitatively was shown.

In the clinical course of RA, there are cases in which joint destruction progresses despite negative CRP tests [25]. This can be explained by persistent localized synovitis in small joints which is not reflected by CRP levels, yet causes progression of joint destruction. Joint ultrasonography is used to evaluate localized inflammation, and is becoming a standard evaluation method in RA [26]. Hammer et al. compared the relationship between joint ultrasonography scores and MRP8/14 in 20 RA patients. They demonstrated a strong correlation between MRP8/14 and ultrasonography scores. On the other hand, Dejaco et al. [27] found no relationship between joint ultrasonography scores and composite measures such as DAS28 or SDAI, although there was a relationship with CRP and morning stiffness. The ultrasonography scores evaluated in this study did not show a significant correlation with either MRP8/14 or disease activity. The difference between our study and the report by Hammer et al. is that while in our study and in the report by Dejaco et al., evaluation of hands and hand joints was conducted, Hammer et al. included large joints such as shoulder joints, elbow joints, and hip joints in their evaluation. Therefore, evaluation limited to the hand joints and fingers was found to be insufficient when joint ultrasono- 
graphy is used for evaluation of RA disease activity. When joint ultrasonography is used for evaluation of systemic synovitis activity due to RA, it may be necessary to select joints to be evaluated and to establish a specific gravity of score by joint size.

In synovitis due to RA, various inflammatory mediators act to induce infiltration of inflammatory cells or proliferation of synovial fibroblasts, however the accurate pathological process, including pathogenesis of RA, has not been completely elucidated. In addition, some of the functions of MRP8/14 in vivo have not been explained. Thus, in order to collect basic data on the relationship between MRP8/14 and disease activity, inflammatory biomarkers, and localized synovitis evaluated using joint ultrasonography in RA patients, patients not using biologic drugs were included in this study. As a result, patients with relatively low disease activity were included and the sample size was relatively small. However, the efficacy of MRP8/14 in evaluating RA disease activity in patients with low disease activity was demonstrated in this study.

This study has some limitations. Currently, methotrexate, steroids, and various biological drugs with different mechanism of action are used in RA treatment; however, the effect of these medications on the dynamic state of MRP8/14 in vivo is unknown. This study was limited to patients who did not use biological drugs, however there were some patients using steroids or immunosuppressive drugs, and these drugs may have affected the results of the study. To analyze the effect of these medications on MRP8/14, additional data collection is considered necessary. It is necessary to establish a more objective method for evaluation of disease activity in RA by combining various medical factors including MRP8/14 in the future.

\section{Conclusion}

The results of this study demonstrated that MRP8/14 correlated with disease activity and serum inflammatory biomarkers in patients not using biological drugs. Therefore, MRP8/14 is considered an effective new method for objective evaluation of synovitis in RA.

\section{Acknowledgements}

This investigator initiated study was supported by a Grant from Pfizer Inc. The sponsors were not involved in the design of the study; enrollment of patients; data collection, analysis, and interpretation; or preparation of the manuscript.

\section{Disclosures}

All authors declare no conflict of interest.

\section{References}

[1] Papes, F., Logan, D.W. and Stowers, L. (2010) The Vomeronasal Organ Mediates Interspecies Defensive Behaviors through Detection of Protein Pheromone Homologs. Cell, 141, 692-703. http://dx.doi.org/10.1016/j.cell.2010.03.037

[2] Lee, S.-M., Vasishtha, M. and Prywes, R. (2010) Activation and Repression of Cellular Immediate Early Genes by Serum Response Factor Cofactors. Journal of Biological Chemistry, 285, 22036-22049.

http://dx.doi.org/10.1074/jbc.M110.108878

[3] Desjardins, J.K. and Fernald, R.D. (2010) What Do Fish Make of Mirror Images? Biology Letters, 6, 744-747. http://dx.doi.org/10.1098/rsbl.2010.0247

[4] Cheng, S.Y. and Delville, Y. (2010) Play Fighting and Corticotropin-Releasing Hormone in the Lateral Septum of Golden Hamsters. Neuroscience, 169, 236-245. http://dx.doi.org/10.1016/j.neuroscience.2010.04.040

[5] De Taboada, L., Yu, J., et al. (2011) Transcranial Laser Therapy Attenuates Amyloid-Beta Peptide Neuropathology in Amyloid-Beta Protein Precursor Transgenic Mice. Journal of Alzheimer's Disease, 23, 521-535.

[6] Krum, S.A., Chang, J., Miranda-Carboni, G. and Wang, C.-Y. (2010) Novel Functions for NFkappaB: Inhibition of Bone Formation. Nature Reviews Rheumatology, 6, 607-611. http://dx.doi.org/10.1038/nrrheum.2010.133

[7] O’Keeffe, S.M., Thome, J. and Coogan, A.N. (2012) The Noradrenaline Reuptake Inhibitor Atomoxetine Phase-Shifts the Circadian Clock in Mice. Neuroscience, 201, 219-230. http://dx.doi.org/10.1016/j.neuroscience.2011.11.002

[8] Shimizu, M., Sakai, H., Shirakami, Y., et al. (2011) Acyclic Retinoid Inhibits Diethylnitrosamine-Induced Liver Tumorigenesis in Obese and Diabetic C57BLKS/J- +(db)/+Lepr(db) Mice. Cancer Prevention Research, 4, 128-136. http://dx.doi.org/10.1158/1940-6207.CAPR-10-0163 
[9] Wu, H., Lin, Y.Y., Li, W.G., et al. (2011) Regulation of Nur77 Expression by Beta-Catenin and Its Mitogenic Effect in Colon Cancer Cells. The FASEB Journal, 25, 192-205. http://dx.doi.org/10.1096/fj.10-166462

[10] Kuribara, M., Kidane, A.H., Vos, G.A.P., et al. (2011) Extracellular-Signal Regulated Kinase Regulates Production of Pro-Opiomelanocortin in Pituitary Melanotroph Cells. Journal of Neuroendocrinology, 23, 261-268. http://dx.doi.org/10.1111/j.1365-2826.2010.02103.x

[11] Kane, D., Roth, J., Frosch, M., et al. (2003) Increased Perivascular Synovial Membrane Expression of Myeloid-Related Proteins in Psoriatic Arthritis. Arthritis \& Rheumatology, 48, 1676-1685. http://dx.doi.org/10.1002/art.10988

[12] Frosch, M., Strey, A., Vogl, T., et al. (2000) Myeloid-Related Proteins 8 and 14 Are Specifically Secreted during Interaction of Phagocytes and Activated Endothelium and Are Useful Markers for Monitoring Disease Activity in Pauciarticular-Onset Juvenile Rheumatoid Arthritis. Arthritis \& Rheumatology, 43, 628-637. http://dx.doi.org/10.1002/1529-0131(200003)43:3<628::AID-ANR20>3.0.CO;2-X

[13] Bakkali-Kassemi, L., El Ouezzani, S., Magoul, R., et al. (2011) Effects of Cannabinoids on Neuropeptide Y and Beta-Endorphin Expression in the Rat Hypothalamic Arcuate Nucleus. British Journal of Nutrition, 105, 654-660. http://dx.doi.org/10.1017/S0007114510004095

[14] Hammer, H.B., Fagerhol, M.K., Wien, T.N. and Kvien, T.K. (2011) The Soluble Biomarker Calprotectin (an S100 Protein) Is Associated to Ultrasonographic Synovitis Scores and Is Sensitive to Change in Patients with Rheumatoid Arthritis Treated with Adalimumab. Arthritis Research \& Therapy, 13, R178. http://dx.doi.org/10.1186/ar3503

[15] Kallupi, M., Cannella, N., Economidou, D., et al. (2010) Neuropeptide S Facilitates Cue-Induced Relapse to Cocaine Seeking through Activation of the Hypothalamic Hypocretin System. Proceedings of the National Academy of Sciences of the United States of America, 107, 19567-19572. http://dx.doi.org/10.1073/pnas.1004100107

[16] Kadar, E., Aldavert-Vera, L., Huguet, G., et al. (2011) Intracranial Self-Stimulation Induces Expression of Learning and Memory-Related Genes in Rat Amygdala. Genes, Brain and Behavior, 10, 69-77. http://dx.doi.org/10.1111/j.1601-183X.2010.00609.x

[17] Lurain, N.S. and Chou, S. (2010) Antiviral Drug Resistance of Human Cytomegalovirus. Clinical Microbiology Reviews, 23, 689-712. http://dx.doi.org/10.1128/CMR.00009-10

[18] Abrams, J.M., Engeland, W.C. and Osborn, J.W. (2010) Effect of Intracerebroventricular Benzamil on Cardiovascular and Central Autonomic Responses to DOCA-Salt Treatment. American Journal of Physiology-Regulatory, Integrative and Comparative Physiology, 299, R1500-R1510. http://dx.doi.org/10.1152/ajpregu.00431.2010

[19] Sunahori, K., Yamamura, M., Yamana, J., et al. (2006) The S100A8/A9 Heterodimer Amplifies Proinflammatory Cytokine Production by Macrophages via Activation of Nuclear Factor Kappa B and p38 Mitogen-Activated Protein Kinase in Rheumatoid Arthritis. Arthritis Research \& Therapy, 8, R69. http://dx.doi.org/10.1186/ar1939

[20] Li, H., Chen, L.Y., Guo, Z., et al. (2012) In Vivo Screening to Determine Neurological Hazards of Nitrogen Dioxide $\left(\mathrm{NO}_{2}\right)$ Using Wistar Rats. Journal of Hazardous Materials, 225-226, 46-53. http://dx.doi.org/10.1016/j.jhazmat.2012.04.063

[21] Hammer, H.B., Odegard, S., Fagerhol, M.K., et al. (2007) Calprotectin (a Major Leucocyte Protein) Is Strongly and Independently Correlated with Joint Inflammation and Damage in Rheumatoid Arthritis. Annals of the Rheumatic Diseases, 66, 1093-1097. http://dx.doi.org/10.1136/ard.2006.064741

[22] Hammer, H.B., Odegard, S., Syversen, S.W., et al. (2010) Calprotectin (a Major S100 Leucocyte Protein) Predicts 10-Year Radiographic Progression in Patients with Rheumatoid Arthritis. Annals of the Rheumatic Diseases, 69, 150-154. http://dx.doi.org/10.1136/ard.2008.103739

[23] Hammer, H.B., Haavardsholm, E.A. and Kvien, T.K. (2008) Calprotectin (a Major Leucocyte Protein) Is Associated with the Levels of Anti-CCP and Rheumatoid Factor in a Longitudinal Study of Patients with Very Early Rheumatoid Arthritis. Scandinavian Journal of Rheumatology, 37, 179-182. http://dx.doi.org/10.1080/03009740701874451

[24] Grippo, A.J., Sgoifo, A., Mastorci, F., et al. (2010) Cardiac Dysfunction and Hypothalamic Activation during a Social Crowding Stressor in Prairie Voles. Autonomic Neuroscience, 156, 44-50. http://dx.doi.org/10.1016/j.autneu.2010.03.003

[25] Ota, K.T., Monsey, M.S., Wu, M.S., et al. (2010) Synaptic Plasticity and NO-cGMP-PKG Signaling Coordinately Regulate ERK-Driven Gene Expression in the Lateral Amygdala and in the Auditory Thalamus Following Pavlovian Fear Conditioning. Learning \& Memory, 17, 221-235. http://dx.doi.org/10.1101/lm.1592510

[26] Tong, J.H., Ng, D.C., Chau, S.L., et al. (2010) Putative Tumour-Suppressor Gene DAB2 Is Frequently Down Regulated by Promoter Hypermethylation in Nasopharyngeal Carcinoma. BMC Cancer, 10, 253. http://dx.doi.org/10.1186/1471-2407-10-253

[27] Dejaco, C., Duftner, C., et al. (2011) Ultrasound-Defined Remission and Active Disease in Rheumatoid Arthritis: Association with Clinical and Serologic Parameters. Seminars in Arthritis and Rheumatism, 41, 761-767. 